9 Whitehouse AM, Duncan JM. Ephedrine psychosis rediscovered. Br J Psychiatry 1987;150:258-61.

10 Pugh CR, Howie SM. Dependence on pseudoephedrine. $\mathrm{Br}$ Psychiatry 1986;149:798.

11 Jelley TM. Phensedyl abuse, Br $\mathcal{F}$ Psychiatry 1987;151:418-9.

12 Lambert T. Paranoid psychoses after abuse of proprietary cold remedies. Br f Psychiatry (in press).

\section{Refeeding hypophosphataemia in anorexia nervosa and alcoholism}

SIR,-The article by Dr Allan D Cumming and colleagues on refeeding hypophosphataemia (22 August, p 490) omits a number of salient points relating to the development of convulsions in alcoholics with complex biochemical disturbances. Several combinations of the abnormalities de scribed might account for the symptoms observed.

Magnesium depletion, in particular, is well known to be a common cause of convulsions in such patients. ${ }^{1}$ In addition, magnesium deficiency induces hypokalaemia and hypocalcaemia, as well as hypomagnesaemia. ${ }^{2}$ In the alcoholic patient described the potential contributions of other ionic imbalances, notably hypokalaemia $(3.2 \mathrm{mmol} / \mathrm{l})$ and hypomagnesaemia $(0.36 \mathrm{mmol} / \mathrm{l})$, are ignored. This is surprising in view of the fact that this patient required "separate infusions of calcium magnesium, and potassium" before recovery.

Though we should not ignore the potential importance of refeeding hypophosphataemia, the contribution of hypophosphataemia itself to the symptoms described remains unclear.

M F RYAN H PANDOV

Department of Clinical Chemistry,

East Birmingham Hospital,

Birmingham B9 5ST

1 Berkelhammer C, Bear RA. A clinical approach to commo electrolyte disorders. Hypomagnesaemia. Can Med Assoc 1985;132:360-8.

2 Brenton DP, Gordon TE. Magnesium. Br f Hosp Med 1984;32. $60-9$

AUTHORS' REPLY,-Drs Ryan and Pandov seem to have misunderstood the purpose of our article which was to emphasise the importance of rapid changes in the plasma phosphate concentration during the refeeding of malnourished patients. Their comments illustrate the confusion that commonly arises between the effects of electrolyte disturbances during chronic nutritional deficiency and acute electrolyte changes after treatment is started. The biochemical variables of our second patient on admission typified those of severe alcoholism: hyponatraemia, hypokalaemia, hypomagnesaemia, and moderate hypophosphataemia. These changes were probably of long standing, and the patient was asymptomatic. Subsequent clinical deterioration after feeding was associated with a decline of plasma phosphate concentration from 0.30 to $0.10 \mathrm{mmol} / \mathrm{l}$, a value indicating severe hypophosphataemia and likely to cause symptoms. Plasma magnesium concentration did not change. As stated in our report, plasma calcium and magnesium concentrations fell during intravenous treatment with phosphate; these are recognised effects of intravenous phosphate administration and were treated as necessary. ${ }^{2}$

Clinically, electrolyte disturbances rarely occur in isolation, and speculation about the relative contribution of each abnormality to the patient's clinical state is seldom helpful. We were careful not to suggest an exclusive causal relation between symptoms and hypophosphataemia, and indeed we commented in the discussion that hypokalaemia, hypomagnesaemia, and acid base disturbance may have contributed. Magnesium depletion is certainly a common feature of chronic alcoholism. We suspect, however, that hypo- magnesaemia was not the primary cause of symptoms in either patient. Neither showed the most common symptoms associated with hypo magnesaemia - paraesthesia, cramp, and tetany. Magnesium deficiency usually induces hypocalcaemia, as Drs Ryan and Pandov comment, ${ }^{4}$ but plasma calcium concentration was normal in both cases. Hypophosphataemia was profound $(<20 \%$ of normal) at the onset of symptoms, with comparatively less depression of plasma magnesium and potassium concentrations. Experimentally, selective phosphate depletion causes magnesuria and hypomagnesaemia ${ }^{35}$; magnesium depletion may cause intracellular phosphate depletion, but the plasma phosphate concentration remains normal.

In relation to the opening sentence by Drs Ryan and Pandov, it should be noted that at no point in our paper do we refer to convulsions in an alcoholic patient. The patient in case 1 suffered from anorexia nervosa alone, and convulsions were not bserved in case 2

We suspect that the clinical importance of changes in the plasma phosphate concentration is not always recognised. We would emphasise that in malnourished patients an "admission blood" will not detect progressive hypophosphataemia during refeeding, and we recommend continued biochemical monitoring until the patient is clinically stable.

Allan D Cumming JACQUELYN R FARQHAR IAN A D BOUCHIER

Edinburgh University Department of Medicine,

Royal Infirmary, Edinburgh EH3 9YW

1 Berkelhammer C, Bear RA. A clinical approach to common electrolyte problems: 3 . Hypophosphatemia. Can Med Assoc $f$ 1984;130:17-23.

Kurokawa K, Levine BS, Lee DBN, Massry SG. Physiology of phosphorus metabolism and pathophysiology of hypophosphatemia and hyperphosphatemia. In: Areiff AI, De Fronzo RA, eds. Fluid, electrolyte and acid base disorders. New York: Churchill Livingstone, 1986.

3 Brenton DP, Gordon TE. Magnesium. Br $\mathcal{F}$ Hosp Med 1984;32: 60-9.

Cronin RE, Knochel JP. Magnesium deficiency. Ado Intern Med 1983;28:509-33.

Massry SG. The clinical syndrome of phosphate depletion. Ado Exp Med Biol 1978;103:301-12.

False positive results of tests for syphilis and outcome of pregnancy

SIR,-Dr J G Thornton and coworkers (8 August, p 354) conclude that pregnant women with a false positive result in one of three non-specific serological tests for syphilis had an increaed rate of pregnancy loss during the first and second trimesters. We are, however, uncertain whether this association is valid.

Only 39 of 71 patients were retested and confirmed as being seropositive, and therefore tech nical false positive reactions were not excluded in the remaining women. "Biological false positive" reactions may be transient or chronic, the latter being present for more than six months. Acute false positive reactions may be associated with infection-for example, herpes simplex virusparticularly when fever is present. Chronic reactions have been reported in intravenous drug users. These maternal conditions may contribute to spontaneous abortion and increased fetal loss. False positive reactions are recognised as a serological abnormality in autoimmune disease, bein more common in women and often predating the clinical manifestations by many years.

Though retrospective matching of the cases was closely related to obstetric performance, there were no reported attempts at matching for ethnic background or social class, which is particularly surprising with regard to ethnic background in view of the higher prevalence of systemic lupus erythematosus in negro women. ${ }^{2}$ We also do not know whether metabolic conditions, such as maternal diabetes mellitus, were taken into account in the study.

Sensitive though these non-specific treponemal tests are, we would argue that they remain an inaccurate screening method for detecting maternal autoimmune disease. The results may be confounded by the exogenous or endogenous factors noted above, just as spontaneous abortions and fetal loss have a multifactorial aetiology.

R LAU

G E FORSTER

The London Hospital (Whitechapel),

London E1 1BB

King A, Nicol C, Rodin P. Venereal diseases. 4th ed. London: Bailliere Tindall, 1980.

Fessel WJ. Systemic lupus erythematosus in the community. Arch Intern Med 1974;134:1027.

Prognosis of subsequent pregnancies after recurrent spontaneous abortion in first trimester

SIR,-The message of the article by Drs Willem Vlaanderen and Pieter E Treffers (11 July, p 92) is that 17 of 21 patients had subsequently successful pregnancies after recurrent spontaneous abortion and that therefore extensive investigation and treatment are unnecessary. Our experience, based on the assessment of over 200 women with a history of recurrent abortion, is quite different.

We agree that the loss of a vital healthy embryo is rare and that abortion in the first trimester is usually preceded by death of the embryo. In our series of 413 first trimester abortions in women who habitually aborted (out of 800 that could be assessed in 186 women), only 31 were abortions of live embryos, 382 being missed abortions. It is unjustified, however, to claim that most aborted fetuses show chromosomal anomalies. Less than $20 \%$ of aborted fetuses may be successfully karyotyped. ${ }^{12}$ Live tissue is necessary for karyotyping, and the necrotic trophoblast obtained from a missed abortion does not usually grow sufficiently in culture to allow karyotyping. It is mere supposition that these abortions have a chromosomal or ovular basis.

Drs Vlaanderen and Treffers conclude that extensive examination is seldom helpful. We agree, but we disagree that recurrent embryonic death in women with a history of recurrent abortion must be regarded as inevitable; it is precisely this type of pregnancy that responds to immunotherapy. Drs Vlaanderen and Treffers quote the results of Mowbray et al: 17 successful pregnancies out of $22 .{ }^{3} \mathrm{We}$ also consistently find that $80 \%$ of subsequent pregnancies progress normally after immunisation. Drs Vlaanderen and Treffers report that 17 of 21 women subsequently had successful pregnancies. Our experience, however, is completely different. Only seven out of 28 women with a history of recurrent abortion who did not receive treatment subsequently had successful pregnancies.

Drs Vlaanderen and Treffers describe patients who had had three or more abortions. Do their suggestions, however, of little investigation and no treatment (apart from reassurance and encouragement) pertain to women who have had five or more abortions?

Our results in women who had had five or more missed abortions in the first trimester (abortions of live embryos are exluded) show that only three of 14 subsequent pregnancies progressed normally, as opposed to 18 of 24 after paternal leucocyte 